KFA-IKP(TH)-1997-19

nucl-th/9801nnn

\title{
Nuclear forces from chiral Lagrangians using the method of unitary transformation I : Formalism
}

\author{
E. Epelbaoum, ${ }^{\dagger \dagger}$ W. Glöckle, ${ }^{\dagger+2}$ Ulf-G. Meißner \\ ${ }^{\ddagger}$ Forschungszentrum Jülich, Institut für Kernphysik (Theorie) \\ D-52425 Jülich, Germany \\ ${ }^{\dagger}$ Ruhr-Universität Bochum, Institut für Theoretische Physik II \\ D-44870 Bochum, Germany
}

\begin{abstract}
We construct the two- and three-nucleon potential based on the most general chiral effective pion-nucleon Lagrangian using the method of unitary transformations. For that, we develop a power counting scheme consistent with this projection formalism. In contrast to previous results obtained in old-fashioned time-ordered perturbation theory, the method employed leads to energy-independent potentials. We discuss in detail the similarities and differences to the existing chiral nucleon-nucleon potentials. We also show that to leading order in the power counting, the three-nucleon forces vanish lending credit to the result obtained by Weinberg using old-fashioned time-ordered perturbation theory.
\end{abstract}

\footnotetext{
\#1 email: evgeni.epelbaum@hadron.tp2.ruhr-uni-bochum.de

\#2 email: walter.gloeckle@hadron.tp2.ruhr-uni-bochum.de

\#3 email: Ulf-G.Meissner@fz-juelich.de
} 


\section{Introduction}

One of the most important and most intensively studied problems in nuclear physics is deriving the forces among nucleons. At present, we are not able to perform direct calculations of these starting from quantum chromodynamics (QCD). Instead of this the alternative approach based on the chiral symmetry of QCD was pioneered by Weinberg [1] [2] and developed by van Kolck et

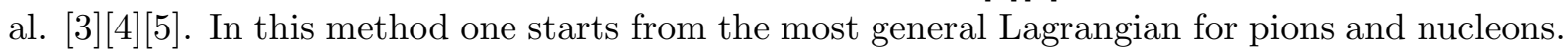
Apart from the usual symmetry constraints, spontaneously broken chiral symmetry is taken into account. Consequently, the couplings of pion fields, which play the rule of Nambu-Goldstone bosons within this formalism, to nucleon fields are of derivative type. The most general pionnucleon Lagrangian is then given by infinite series of terms with increasing number of derivatives and nucleon fields. Explicit breaking of chiral symmetry, leading to the nonvanishing but small pion mass, can also be treated systematically.

The Lagrangian constructed in this way can then be used to derive the expansion of low energy S-matrix elements in powers of some low energy scale $Q$. To be more precise, the 4-momenta of the external pions and the 3-momenta of the external nucleons must be no larger but of the same order as $Q$. It is therefore natural to treat the nucleons nonrelativistically. More precisely, the dimensionless expansion parameter is $Q / \Lambda_{\chi}$, with $\Lambda_{\chi}$ the scale of the spontaneous symmetry violation. Although the value of $\Lambda_{\chi}$ is not precisely known, it is related to the scale of the higher mass states in QCD, i.e. $\Lambda_{\chi}$ can be chosen to be the mass of the rho or $2 \pi F_{\pi} \simeq 1.2 \mathrm{GeV}$, with $F_{\pi}=186 \mathrm{MeV}$ the weak pion decay constant. All other degrees of freedom such as higher mass mesons, antinucleons or other baryons are less relevant for low-energy physics and may be considered as to be integrated out of the theory. The information about them is then hidden in parameters of the Lagrangian. Only the $\Delta(1232)$ resonance, which has a mass close to the one of the nucleon and is strongly coupled to the pion-nucleon system, is sometimes kept explicitly. Once the effective Lagrangian is constructed, one can formulate the power counting rules in order to determine the power of $Q$ for any given Feynmann diagram. It was shown in [6] that chiral symmetry guarantees a perturbative expansion of low-energy processes because the power of $Q$ for any given process is bounded from below. In particular, only a finite number of tree and loop diagrams can contribute to given processes at any finite order.

This scheme works fairly well for purely pionic or processes with only one nucleon [7] [8]. However, complications arise when one tries to treat processes with few nucleons caused by infrared divergences (in the static approximation for the nucleons), which destroy the power counting. These difficulties are in principle to be expected: the presence of low-energy bound states signals the failure of perturbation theory. One way of solving this problem, first introduced by Weinberg [1], is to use the time-dependent ("old-fashioned") perturbation theory instead of the covariant one. The expansion of the S-matrix, obtained using this formalism, has the form of a Lippmann-Schwinger equation with an effective potential, defined as a sum of all diagrams without pure nucleonic intermediate states. Such states would lead in "old-fashioned" perturbation theory to energy denominators, which are by a factor of $Q / m_{N}$ smaller then those from the states with pions and which destroy the power counting. The effective potential is free from such small energy denominators and can in principle be calculated perturbativly to any given precision. Many other equivalent schemes are known leading to same results like that due to Bloch and Horowitz [9] or the Tamm-Dancoff approximation [10]. The effective potential, derived using the old-fashioned time-ordered perturbation theory, possesses one unpleasant property: it is in general explicitly dependent on the energy of incoming nucleons and as a consequence of this is not Hermitean. Furthermore, the nucleonic wave functions are not orthonormal in this

\footnotetext{
${ }^{\# 4}$ Note that for easier comparison with the existing literature, we use this particular normalization of $F_{\pi}$.
} 
approach [1].

One can avoid these problems by using the method of unitary transformation. It was already applied succesfully in cases where one has an expansion in a coupling constant, such as the pion-nucleon coupling, see e.g. refs. [12]. Our aim is to show how to apply this method to the case of chiral perturbation theory for pions and nucleons, in which the small momenta of external particles play the role of the expansion parameter. In fact, our considerations are more general since they can be applied to any effective field theory of Goldstone bosons coupled to some massive matter fields.

In section 2 we give a short introduction to the Bloch-Horowitz scheme and the method of unitary transformations as pionneered by Okubo [15] and by Fukuda et al. [16]. In section 3, we show how to apply this formalism to the chiral invariant effective Lagrangian for pions and nucleons. We use these ideas in section 4 in order to calculate the two-nucleon force in leading and next-to-leading order using the same effective Lagrangian as in [4] and compare our results with those obtained using "old-fashioned" perturbation theory. We also derive the leading order three-nucleon force and shows that it vanishes, a result first found by Weinberg [1]. Section 5 contains the summary and conclusions. Various technicalities are relegated to the appendices.

\section{Bloch-Horowitz scheme and the method of unitary transfor- mation}

A good introduction to both schemes is given in [11. We briefly repeat the main points, mostly to establish our notation and keep the paper self-contained. Furthermore, we need the basic equations to develop the chiral power counting scheme in the following.

We start from the Hamiltonian

$$
H=H_{0}+H_{I}
$$

for pions and nucleons with $H_{0}\left(H_{I}\right)$ denoting the free (interaction) part. The whole problem for an arbitrary number of interacting particles can be cast in the form of a Schrödinger equation,

$$
H|\Psi\rangle=E|\Psi\rangle
$$

In order to solve this equation for nucleon-nucleon scattering, it is advantageous to project it onto a subspace $|\phi\rangle$ of the full Fock space $|\Psi\rangle$ which contains only nucleonic states. We shall denote the remaining part of the Fock space by $|\psi\rangle:|\Psi\rangle=|\phi\rangle+|\psi\rangle$. Let $\eta$ and $\lambda$ be projection operators on the states $|\phi\rangle$ and $|\psi\rangle$ which satisfy $\eta^{2}=\eta, \lambda^{2}=\lambda, \eta \lambda=\lambda \eta=0$ and $\lambda+\eta=\mathbf{1}$. Eq. (2.2) can now be written in the form

$$
\left(\begin{array}{cc}
\eta H \eta & \eta H \lambda \\
\lambda H \eta & \lambda H \lambda
\end{array}\right)\left(\begin{array}{l}
|\phi\rangle \\
|\psi\rangle
\end{array}\right)=E\left(\begin{array}{c}
|\phi\rangle \\
|\psi\rangle
\end{array}\right)
$$

In the Bloch-Horowitz scheme [9] one obtains from the second line of this equation an expression for the state $|\psi\rangle$,

$$
|\psi\rangle=\frac{1}{E-\lambda H \lambda} \lambda H \eta|\Psi\rangle
$$

which can then be used to reduce the first line of eq. (2.3) to

$$
\left(H_{0}+V_{\text {eff }}(E)\right)|\phi\rangle=E|\phi\rangle
$$


with an effective potential $V_{\text {eff }}(E)$ given by

$$
V_{\text {eff }}(E)=\eta H_{I} \eta+\eta H_{I} \lambda \frac{1}{E-\lambda H \lambda} \lambda H_{I} \eta
$$

Expanding the denominator in eq. (2.6) in powers of $H_{I}$ leads to

$$
V_{\mathrm{eff}}(E)=\eta H_{I} \eta+\sum_{n=0}^{\infty} \eta H_{I} \lambda \frac{1}{E-H_{0}}\left(\lambda H_{I} \lambda \frac{1}{E-H_{0}}\right)^{n} \lambda H_{I} \eta .
$$

In this form it is obviously identical with the result obtained from "old-fashioned" perturbation theory [13] [14].

The effective Hamiltonian for nucleons can also be derived from eq. (2.3) in another way, which we shall refer to as the method of unitary transformation. Introducing new states $|\chi\rangle$ and $|\varphi\rangle$, which are related to $|\phi\rangle$ and $|\psi\rangle$ by the unitary transformation

$$
\left(\begin{array}{l}
|\chi\rangle \\
|\varphi\rangle
\end{array}\right)=U^{\dagger}\left(\begin{array}{l}
|\phi\rangle \\
|\psi\rangle
\end{array}\right)
$$

one can rewrite eq. (2.3) in an equivalent form

$$
U^{\dagger} H U\left(\begin{array}{l}
|\chi\rangle \\
|\varphi\rangle
\end{array}\right)=E\left(\begin{array}{c}
|\chi\rangle \\
|\varphi\rangle
\end{array}\right)
$$

We can decouple the two subspaces $|\chi\rangle$ and $|\varphi\rangle$ by choosing $U$ such that the operator $U^{\dagger} H U$ is diagonal. We adopt the ansatz of Okubo [15] for $U$. It has the form

$$
U=\left(\begin{array}{cc}
\left(1+A^{\dagger} A\right)^{-1 / 2} & -A^{\dagger}\left(1+A A^{\dagger}\right)^{-1 / 2} \\
A\left(1+A^{\dagger} A\right)^{-1 / 2} & \left(1+A A^{\dagger}\right)^{-1 / 2}
\end{array}\right)
$$

with the operator $A$ satisfying

$$
A=\lambda A \eta .
$$

The requirement for $U^{\dagger} H U$ to be diagonal leads to the following nonlinear equation for $A$ :

$$
\lambda(H-[A, H]-A H A) \eta=0 .
$$

In the case when the interaction Hamiltonian $H_{I}$ can be treated as a small perturbation, it is possible to solve Eq. (2.12) perturbatively to any order. For instance for the Hamiltonian $H$ represented by

$$
H=H_{0}+\sum_{n=1}^{\infty} H_{n}
$$

with the index $n$ denoting the power of the coupling constant, one assumes the operator $A$ to be of the form

$$
A=\sum_{n=1}^{\infty} A_{n}
$$

The solution of eq. (2.12) to order $n$ is then given by

$$
A_{n}=\frac{1}{\mathcal{E}-H_{0}} \lambda\left\{H_{n}+\sum_{i=1}^{n-1} H_{i} A_{n-i}-\sum_{i=1}^{n-1} A_{n-i} H_{i}-\sum_{i=1}^{n-2} \sum_{j=1}^{n-j-1} A_{i} H_{j} A_{n-i-j}\right\} \eta \text {. }
$$


Here, we denote the free-particle energy of the state $|\eta\rangle$ by $\mathcal{E}$. One can see from eq. 2.15) that it is possible to find $A_{n}$ for every $n$ recursively, starting from $A_{1}$. The effective Hamiltonian, which operates solely in the subspace $|\chi\rangle$, is given by

$$
H_{\mathrm{eff}}=\eta\left(1+A^{\dagger} A\right)^{-1 / 2}\left(H+A^{\dagger} H+H A+A^{\dagger} H A\right)\left(1+A^{\dagger} A\right)^{-1 / 2} \eta,
$$

as it follows from eq. (2.9). Expanding $\left(1+A^{\dagger} A\right)^{-1 / 2}$ and using eqs. (2.13), (2.14), (2.15) one can obtain the effective Hamiltonian to any order in the coupling constant.

Several modifications are necessary by applying the formalism described above to effective Lagrangians (Hamiltonians) and in particular to chiral invariant Lagrangians. First, the expansion in powers of a coupling constant must be replaced by the expansion in powers of small momenta. For doing that, power counting rules are necessary. Furthermore, one expects the operator $\lambda A \eta$ to consist of an infinite number of terms to any order of $Q$ caused by infinite number of vertices in the Hamiltonian. We now show how these problems can be solved.

\section{Application to chiral invariant Hamiltonians}

We want first to recall the structure of the most general chiral invariant Hamilton density for pions and nucleons,

$$
\mathcal{H}=\mathcal{H}_{0}+\mathcal{H}_{I}
$$

We shall treat the nucleons nonrelativistically as it also has been done in [盺运]目. The purely nucleonic part of $\mathcal{H}_{0}$ is nothing but the kinetic energy

$$
\mathcal{H}_{N_{0}}=-N^{\dagger} \frac{\vec{\nabla}^{2}}{2 m} N
$$

where $m$ denotes the nucleon mass. The mass term disappears from the Hamiltonian when one separates the basic time-dependence of nucleon fields (in the rest-frame) via

$$
N=\mathrm{e}^{-i m t} \tilde{N}
$$

In higher orders in small momenta, which we shall not treat here, relativistic corrections to eq. (3.2) must be taken into account and the free nucleonic Hamilton density looks like

$$
\mathcal{H}_{N_{0}}=\mathcal{H}_{N_{0}}^{(2)}+\mathcal{H}_{N_{0}}^{(4)}+\mathcal{H}_{N_{0}}^{(6)}+\ldots
$$

with $\mathcal{H}_{N_{0}}^{(2)}$ given by eq. (3.2). The upper indices denote the number of derivatives acting on $N$. These terms stem from the expansion of the relativistic kinetic energy in powers of $1 / 2 \mathrm{~m}$. A path integral approach to derive such relativistic corrections explicitly from the underlying relativistic theory in the one-baryon sector is spelled out in [18]. The free Hamilton density for pion fields is given by

$$
\mathcal{H}_{\pi 0}=\frac{1}{2} \dot{\pi}^{2}+\frac{1}{2}(\vec{\nabla} \boldsymbol{\pi})^{2}+\frac{1}{2} m_{\pi}^{2} \boldsymbol{\pi}^{2},
$$

where the ${ }^{\prime .}$, denotes the time derivative and $m_{\pi}$ the pion mass. We split the interaction Hamilton density into three parts:

$$
\mathcal{H}_{I}=\mathcal{H}_{\pi \pi}+\mathcal{H}_{N N}+\mathcal{H}_{\pi N} .
$$

The first piece describes self-interactions of pions and contains an even number of derivatives and pion field operators and any number of $m_{\pi}^{2}$-factors. The terms with two derivatives (or one $m_{\pi}^{2}$ ) have at least four pion fields. 
The second piece in eq. (3.6) consists of four or more nucleon fields and any number of derivatives. The terms of $\mathcal{H}_{\pi N}$ have any number of pion fields and either two nucleon fields and at least one derivative (or $m_{\pi}^{2}$ ) or four or more nucleon fields and any number of derivatives and factors of $m_{\pi}$. The structure of the effective Hamiltonian is now clarified and the explicit form we use is given in Appendix C. Because of baryon number conservation and the absence of antinucleons, the subspaces of the Fock space with different number of nucleons are automatically decoupled. That is why we define the state $|\phi\rangle$ as consisting of $N$ nucleons only. The state $|\psi\rangle$ contains then $N$ nucleons and at least one pion. To avoid difficulties with an infinite number of vertices in the Hamiltonian, we shall split the subspace $|\psi\rangle$ into a series of orthonormal subspaces with definite numbers of pions

$$
|\psi\rangle=\left|\psi^{1}\right\rangle+\left|\psi^{2}\right\rangle+\left|\psi^{3}\right\rangle+\ldots
$$

The indices denote the number of pions. Analogously we introduce the corresponding projectors

$$
\lambda=\lambda^{1}+\lambda^{2}+\lambda^{3}+\ldots
$$

with the properties

$$
\lambda^{i} \lambda^{j}=\delta_{i j} \lambda^{i}
$$

Using eq. (3.9) we can rewrite eq. (2.12) in the form of an infinite system of equations

$$
\begin{aligned}
& \lambda^{1}(H-[A, H]-A H A) \eta=0 \\
& \lambda^{2}(H-[A, H]-A H A) \eta=0 \\
& \ldots \\
& \lambda^{i}(H-[A, H]-A H A) \eta=0 \\
& \ldots
\end{aligned}
$$

Because of the property eq. 2.11) of the operator $A$ each single equation in eqs. (3.10) can be expressed as

$$
\lambda^{i} H_{I} \eta+\sum_{j=1}^{\infty} \lambda^{i} H_{I} \lambda^{j} A \eta+\lambda^{i} H_{0} \lambda^{i} A \eta-\lambda^{i} A \eta H_{I} \eta-\lambda^{i} A \eta H_{0} \eta-\sum_{j=1}^{\infty} \lambda^{i} A \eta H_{I} \lambda^{j} A \eta=0
$$

To solve the system of eqs. (3.10), we make use of the usual philosophy of effective theories. We are interested only in low-energy processes. When the renormalization scale for loops is chosen to be of the same order as the low-energy scale $Q$, it is the only quantity with dimensionality of energy apart from the coupling constants. The low-energy matrix elements can then be classified by powers of $Q$ by use of simple dimensional analysis. This immediately raises the following question: The projection of the Hamiltonian in eq. (2.16) involves (in principle) an integration over all momenta, whereas the matrix elements of the operator $A$, which can be found from eq. (3.10), are obtained from a low-energy expansion. Does this amount to a consistent procedure? The answer to this is yes, because of the renormalization, after which all typical momenta in virtual processes are bounded and of the order of $Q$.

Now we make an ansatz for the operator $\lambda^{i} A \eta$, which will be justified at the end of this section: we assume that it consists of equal number of vertices from $H_{I}$ and energy denominators. Each of these denominators contributes a factor of $1 / Q$. It is now easy to calculate the power $\nu_{A}$ of $Q$ of the matrix element $\left\langle\psi^{i}|A| \phi\right\rangle$ using the usual topological identities,

$$
\left\langle\psi^{i}|A| \phi\right\rangle=Q^{\nu_{A}} \mathcal{F}\left(Q / \Lambda_{\chi}, \mu / \Lambda_{\chi}, g_{i}\right)
$$


with $\mu$ the regularization scale (since regularization is needed to render certain loop integrals finite) and $g_{i}$ collectively denoting all coupling constants (low-energy constants). Each integration over virtual momenta brings three powers and each derivative one power of $Q$, respectively. The pionic phase-space factors $1 / \sqrt{2 \omega}$, the energy denominators and $\delta$-functions give each a factor of $Q^{-1 / 2}, Q^{-1}$ and $Q^{-3}$, in order. For $L$ loops, $C$ separately connected pieces, $I_{p}$ internal pion lines and $V_{i}$ vertices of type $i$ with $d_{i}$ derivatives one finds

$$
\nu_{A}=3 L+\sum_{i} V_{i} d_{i}-I_{p}-\sum_{i} V_{i}-3(C-1)
$$

According to our assumption $\sum_{i} V_{i}$ stands for the number of energy denominators. Here and in what follows we do not count the overall $\delta$-function and the phase-space factors of the external pions. Now we use the general identities

$$
\begin{gathered}
L=C+I_{p}+I_{n}-\sum_{i} V_{i}, \\
2 I_{n}+E_{n}=\sum_{i} V_{i} n_{i},
\end{gathered}
$$

and

$$
2 I_{p}+E_{p}=\sum_{i} V_{i} p_{i}
$$

where $E_{n}=2 N$ is the number of external nucleon lines, $E_{p}$ the number of external pion lines, $I_{n}$ the number of internal nucleon lines and $n_{i}\left(p_{i}\right)$ the number of nucleon (pion) fields at a vertex of type $i$, to cast eq. (3.13) into the form

$$
\nu_{A}=3-3 N-E_{p}+\sum_{i} V_{i} \kappa_{i}
$$

with

$$
\kappa_{i}=d_{i}+\frac{3}{2} n_{i}+p_{i}-4
$$

Again we point out that this result takes its form due to the ansatz that the projected operators $\lambda^{i} A \eta$ with a given number of nucleons and pions consist of an equal number of vertices and energy denominators. Also, it should be mentioned that we use the number of external pion lines to express the counting index $\nu_{A}$ instead of the number of loops and of separately connected pieces as it has been done in [1]-[5]. This is more natural in the projection formalism employed here. Let us take a closer look at vertex dimension $\kappa_{i}$, which is related to the canonical field dimension. It is well known that all interactions can be classified with respect to $\kappa_{i}$. Those with $\kappa_{i}<0$ are called relevant, with $\kappa_{i}=0$ marginal and with $\kappa_{i}>0$ irrelevant. The last ones are "harmless" and can be well treated within low-energy effective field theories. In contrast to these, the relevant and marginal interactions lead to complications with the power counting. This becomes immediately clear when one takes a look at eq. (3.17): the number of possible diagrams for a given process and to a given order is no more bounded. Furthermore, for relevant interactions the number $\nu_{A}$ is even not bounded from below. That is why the perturbative treatment is not possible in this case. For more details about the role of such interactions in effective field theories see [19]. Chiral symmetry does not allow any relevant or marginal interactions in the interaction Hamiltonian $H_{I}$. The minimum possible value of $\kappa_{i}$ for vertices in $H_{I}$ is one. The corresponding vertex contains two nucleons, one pion and one derivative. In general, for vertices with two nucleons and $p_{i}$ pions one can easily find that $\kappa_{i} \geq p_{i}$. The interactions with pions only have the vertex dimension $\kappa_{i} \geq 2$ with $\kappa_{i}=2$ corresponding to 
the interaction with four pion fields and two derivatives. Again, for those vertices with an even number $p_{i}$ of pions and without nucleons it follows, that $\kappa_{i} \geq \max \left(2,-2+p_{i}\right)$. Using eq. (3.17) one can determine the minimum value of $\nu_{A}$ for $\lambda^{4 k+i} A \eta$ with $k$ positive integer or zero and $i=0,1,2,3$ :

$$
\min \left(\nu_{A}\right)=3-3 N-2 k
$$

All diagrams corresponding to such operators consist of maximum possible number of pure pionic vertices with $p_{i}$ pion fields and $\kappa_{i}=-2+p_{i}$ and any number of interactions with two nucleons, $p_{i}$ pions and $\kappa_{i}=p_{i}$ without intermediate pion states. In the case $i=3$ one intermediate pion state may also occur.

We define the order of the operator $\lambda^{4 k+i} A \eta$ by the number $l(l=0,1,2,3, \ldots)$ which satisfies

$$
\nu_{A}=3-3 N-2 k+l,
$$

and introduce the following notation: $\lambda^{4 k+i} A_{l} \eta$. Let us now find the power $\nu$ of $Q$ for every term in eq. (3.11). By $H_{\kappa}$ we denote a vertex from the interaction Hamiltonian $H_{I}$, with the index $\kappa$ given by eq. (3.18). Details are relegated to appendix A. It can be seen from eqs. (A.7), (A.10), (A.12), (A.15) and (A.16) that the minimum possible value of $\nu$ for eq. (3.11) is given by

$$
\min (\nu)=4-3 N-2 k \quad .
$$

We now show how to solve the system of eqs. (3.10) perturbatively. For that, we define the order of eq. (3.11) to be $r \geq 0$ given by

$$
r=\nu-(4-3 N-2 k)
$$

In appendix B we check what kind of operators $\lambda^{4 k+i} A_{l} \eta$ enter the eq. (3.11) at each order $r$. That equation can be expressed as

$$
E\left(\lambda^{4 k+i}\right) \lambda^{4 k+i} A_{l=r} \eta=-\lambda^{4 k+i}\{H-[A, H]-A H A\} \eta .
$$

The free energy is always denoted by $E$. In this case it is the pionic part only. In the curly brackets we have written in symbolic form all remaining terms of eq. (3.11). For $i=1,2$ they contain only operators of the type $\lambda^{4 \tilde{k}+\tilde{i}} A_{l} \eta$ with

$$
4 \tilde{k}+\tilde{i}<4 k+i \quad \text { for } \quad l=r, \quad \text { or } \quad l<r .
$$

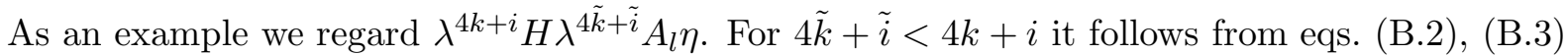
that $l \leq r$. For $4 \tilde{k}+\tilde{i}=4 k+i$, eq. (B.4) leads to $l \leq r-2$. Finally for $4 \tilde{k}+i>4 k+i$ eqs. (B.5)-(B.8) require $l \leq r-2$. Eq. (3.24) is also true for $i=3$ apart from the single term $-\lambda^{4 k+3} H_{1} \lambda^{4(k+1)} A_{l=r} \eta$. This is due to eq. (B.7). In the case $i=0$, only the operators $\lambda^{4 \tilde{k}+\tilde{i}} A_{l} \eta$, restricted by the conditions

$$
\tilde{i}=0, \quad \tilde{k}<k \quad \text { for } \quad l=r, \quad \text { or } \quad l<r,
$$

can enter the right hand side of eq. (3.23). In all cases the value of $\tilde{k}$ is bounded from above by the inequality

$$
\tilde{k} \leq k+\frac{1}{6}(r-l+8) .
$$

as can be defered from the second inequality in $(\overline{B .8})$ and the equality in (B.16). Now it is clear how to deal with the system of equations (3.10). At each order $r$ one starts by solving 
the equations with $i=0$ and with increasing number $k$ to define all operators $\lambda^{4 k} A_{l=r} \eta$. This requires knowledge of operators $\lambda^{4 \tilde{k}} A_{l} \eta$ of the same order $l=r$ with $\tilde{k}<k$ and a finite number of operators in lower orders $l<r$. The next step is to solve the remaining equations with $i=1,2,3$ with increasing number $4 k+i$ starting from $4 k+i=1$. After that one can go to the next order $r+1$. The number of equations to be solved in each order can be estimated by use of eq. (3.26) and the inequalities of appendix B.

To justify our ansatz about the structure of the operator $A$, consider the starting equations at order $r=0$, given by

$$
\begin{aligned}
& E\left(\lambda^{4}\right) \lambda^{4} A_{0} \eta=\lambda^{4} H_{2} \eta, \\
& E\left(\lambda^{1}\right) \lambda^{1} A_{0} \eta=\lambda^{1} H_{1} \eta,
\end{aligned}
$$

where $E\left(\lambda^{i}\right)$ corresponds to the kinetic energy of the pions in the state $\lambda^{i}$. From eq. (3.23) one can see that the unknown operator $\lambda^{4 k+i} A_{l=r} \eta$ has the structure which we assumed at the beginning of this section, if and only if the already known operators $\lambda A \eta$ entering the right hand side of this equation have precisely this form. Therefore, to proof our ansatz recursively for all operators $\lambda A \eta$ it is sufficient to see that it holds for the corresponding starting operators in eqs. (3.27) and (3.28), which is obviously the case.

\section{Two- and three-nucleon forces using the method of unitary transformation}

We shall now practically apply the formalism described in the last section and derive an effective Hamiltonian for nucleons in leading and next-to-leading orders. As a starting point we use the effective chiral invariant Hamiltonian for nucleons and pions, which was specified at the beginning of the last section. Its explicit form is given in appendix $\mathrm{C}$.

\subsection{Two and many nucleon potential in the projection formalism}

First of all we look at the eq. (2.16) and define the order of all terms on its right hand side. Introducing the operators

$$
L_{t} \equiv \eta A_{l_{t}}^{\dagger} \lambda^{4 k_{t}+i_{t}} A_{l^{\prime}{ }_{t}} \eta
$$

and its chiral power (the power of $Q$ )

$$
\nu_{t}=3-3 N+\tilde{\nu}_{t}
$$

with

$$
\tilde{\nu}_{t}=4 k_{t}+2 i_{t}+l_{t}+l_{t}^{\prime},
$$

evaluated via eqs. (3.17) and (A.1) one gets contributions of the following types. In the following $\nu$ denotes the corresponding power of $Q$.

$$
\begin{aligned}
& {\left[\prod_{t} L_{t}\right] } \\
& \nu=3-3 N+\sum_{t} \tilde{\nu}_{t} \\
\text { 2. } & {\left[\prod_{t} L_{t}\right] \eta H_{\kappa} \eta\left[\prod_{s} L_{s}\right] }
\end{aligned}
$$




$$
\begin{gathered}
\nu=4-3 N+\kappa+\sum_{t} \tilde{\nu}_{t}+\sum_{s} \tilde{\nu}_{s} \\
\text { 3. } \quad\left[\prod_{t} L_{t}\right] \eta A_{l_{m}}^{\dagger} \lambda^{4 k_{m}+i_{m}} H_{\kappa} \eta\left[\prod_{s} L_{s}\right] \\
\text { and } \quad\left[\prod_{t} L_{t}\right] \eta H_{\kappa} \lambda^{4 k_{m}+i_{m}} A_{l_{m}} \eta\left[\prod_{s} L_{s}\right] \\
\nu=4-3 N+\kappa+2 k_{m}+i_{m}+l_{m}+\sum_{t} \tilde{\nu}_{t}+\sum_{s} \tilde{\nu}_{s} \\
\quad\left[\prod_{t} L_{t}\right] \eta A_{l_{m}}^{\dagger} \lambda^{4 k_{m}+i_{m}} H_{\kappa} \lambda^{4 k_{n}+i_{n}} A_{l_{n}} \eta\left[\prod_{s} L_{s}\right] \\
\quad \nu=4-3 N+\kappa+l_{m}+l_{n}+2 k_{m}+2 k_{n}+i_{m}+i_{n}+\sum_{t} \tilde{\nu}_{t}+\sum_{s} \tilde{\nu}_{s} .
\end{gathered}
$$

In case of the unit operator in eq. (2.16) one has to drop the $\tilde{\nu}$ 's. The effective potential can be easily read off from the effective Hamiltonian via

$$
V_{\text {eff }}=H_{\text {eff }}-H_{0}
$$

From now, we denote the contribution from the free Hamiltonian $H_{0}$ by the pertinent nucleonic and pionic free energies, $\mathcal{E}$ and $\omega$, respectively. Using now eqs. (2.16) and (4.4) - 4.7) one can write the leading term of order $\nu=6-3 N$ of the $N$-body potential as:

$$
V_{\mathrm{eff}}^{(6-3 N)}=\eta\left(H_{2}+A_{0}^{\dagger} \lambda^{1} H_{1}+H_{1} \lambda^{1} A_{0}+A_{0}^{\dagger} \lambda^{1} \omega A_{0}\right) \eta
$$

Note that in the last term only the pionic free energy $\omega$ contributes in lowest order. As noted in [2] and [3], there are is contribution to the potential to order $7-3 N$ because of parity invariance. Another way to see this is the fact that there is no nonvanishing operator $\lambda^{1} H_{2} \eta$. At next order, $8-3 N$, one gets a more complicated expression for the potential:

$$
\begin{aligned}
V_{\mathrm{eff}}^{(8-3 N)=} & \eta\left(H_{4}+A_{2}^{\dagger} \lambda^{1} H_{1}+A_{0}^{\dagger} \lambda^{1} H_{3}+H_{3} \lambda^{1} A_{0}+H_{1} \lambda^{1} A_{2}\right. \\
& +A_{0}^{\dagger} \lambda^{1} H_{2} \lambda^{1} A_{0}-\frac{1}{2} A_{0}^{\dagger} \lambda^{1} A_{0} \eta H_{2}-\frac{1}{2} H_{2} \eta A_{0}^{\dagger} \lambda^{1} A_{0} \\
& +A_{0}^{\dagger} \lambda^{1} \mathcal{E} A_{0}-\frac{1}{2} A_{0}^{\dagger} \lambda^{1} A_{0} \mathcal{E}-\frac{1}{2} \mathcal{E} A_{0}^{\dagger} \lambda^{1} A_{0}+A_{2}^{\dagger} \lambda^{1} \omega A_{0}+A_{0}^{\dagger} \lambda^{1} \omega A_{2} \\
& +A_{0}^{\dagger} \lambda^{1} H_{1} \lambda^{2} A_{0}+A_{0}^{\dagger} \lambda^{2} H_{1} \lambda^{1} A_{0} \\
& -\frac{1}{2} A_{0}^{\dagger} \lambda^{1} A_{0} \eta A_{0}^{\dagger} \lambda^{1} H_{1}-\frac{1}{2} A_{0}^{\dagger} \lambda^{1} A_{0} \eta H_{1} \lambda^{1} A_{0}-\frac{1}{2} A_{0}^{\dagger} \lambda^{1} H_{1} \eta A_{0}^{\dagger} \lambda^{1} A_{0} \\
& -\frac{1}{2} H_{1} \lambda^{1} A_{0} \eta A_{0}^{\dagger} \lambda^{1} A_{0}-\frac{1}{2} A_{0}^{\dagger} \lambda^{1} A_{0} \eta A_{0}^{\dagger} \lambda^{1} \omega A_{0}-\frac{1}{2} A_{0}^{\dagger} \lambda^{1} \omega A_{0} \eta A_{0}^{\dagger} \lambda^{1} A_{0} \\
& \left.+A_{0}^{\dagger} \lambda^{2} H_{2}+H_{2} \lambda^{2} A_{0}+A_{0}^{\dagger} \lambda^{2}\left(\omega_{1}+\omega_{2}\right) A_{0}\right) \eta .
\end{aligned}
$$

Note that the $\mathcal{E}$ 's denote the nucleonic free energies related to the accompanying projection operators $\left(\lambda\right.$ or $\eta$ ). The operators $\lambda^{1} A_{0} \eta, \lambda^{2} A_{0} \eta, \lambda^{1} A_{2} \eta$ can be evaluated along the lines described in the last section. This leads to:

$$
\begin{aligned}
\lambda^{1} A_{0} \eta & =-\frac{\lambda^{1}}{\omega} H_{1} \eta \\
\lambda^{2} A_{0} \eta & =-\frac{\lambda^{2}}{\omega_{1}+\omega_{2}} H_{2} \eta-\frac{\lambda^{2}}{\omega_{1}+\omega_{2}} H_{1} \lambda^{1} A_{0} \eta,
\end{aligned}
$$




$$
\begin{aligned}
\lambda^{1} A_{2} \eta= & -\frac{\lambda^{1}}{\omega} H_{3} \eta-\frac{\lambda^{1}}{\omega} H_{1} \lambda^{2} A_{0} \eta-\frac{\lambda^{1}}{\omega} H_{2} \lambda^{1} A_{0} \eta+\frac{\lambda^{1}}{\omega} A_{0} \eta H_{1} \lambda^{1} A_{0} \eta \\
& +\frac{\lambda^{1}}{\omega} A_{0} \eta H_{2} \eta-\mathcal{E} \frac{\lambda^{1}}{\omega} A_{0} \eta+\frac{\lambda^{1}}{\omega} A_{0} \eta \mathcal{E} .
\end{aligned}
$$

Putting eqs. (4.11) - (4.13) into eqs. (4.9) and 4.10) and performing straightforward algebraic manipulations, one obtains the potential expressed as

$$
\begin{aligned}
V_{\mathrm{eff}}^{(6-3 N)=} & \eta\left(H_{2}-H_{1} \frac{\lambda^{1}}{\omega} H_{1}\right) \eta \\
V_{\mathrm{eff}}^{(8-3 N)}= & \eta\left(H_{4}-H_{1} \frac{\lambda^{1}}{\omega} H_{3}-H_{3} \frac{\lambda^{1}}{\omega} H_{1}\right. \\
& +H_{1} \frac{\lambda^{1}}{\omega} H_{2} \frac{\lambda^{1}}{\omega} H_{1}+H_{1} \frac{\lambda^{1}}{\omega} H_{1} \frac{\lambda^{2}}{\omega_{1}+\omega_{2}} H_{2}+H_{2} \frac{\lambda^{2}}{\omega_{1}+\omega_{2}} H_{1} \frac{\lambda^{1}}{\omega} H_{1} \\
& -H_{2} \frac{\lambda^{2}}{\omega_{1}+\omega_{2}} H_{2} \\
& -H_{1} \frac{\lambda^{1}}{\omega} H_{1} \frac{\lambda^{2}}{\omega_{1}+\omega_{2}} H_{1} \frac{\lambda^{1}}{\omega} H_{1}+\frac{1}{2} H_{1} \frac{\lambda^{1}}{(\omega)^{2}} H_{1} \eta H_{1} \frac{\lambda^{1}}{\omega} H_{1}+\frac{1}{2} H_{1} \frac{\lambda^{1}}{\omega} H_{1} \eta H_{1} \frac{\lambda^{1}}{(\omega)^{2}} H_{1} \\
& -\frac{1}{2} H_{1} \frac{\lambda^{1}}{(\omega)^{2}} H_{1} \eta H_{2}-\frac{1}{2} H_{2} \eta H_{1} \frac{\lambda^{1}}{(\omega)^{2}} H_{1} \\
& \left.+H_{1} \mathcal{E} \frac{\lambda^{1}}{(\omega)^{2}} H_{1}-\frac{1}{2} \mathcal{E} H_{1} \frac{\lambda^{1}}{(\omega)^{2}} H_{1}-\frac{1}{2} H_{1} \frac{\lambda^{1}}{(\omega)^{2}} H_{1} \mathcal{E}\right) \eta .
\end{aligned}
$$

Consider first the leading order potential, eq. (4.14). It consists of two terms, the one-pion exchange $\sim H_{1}\left(\lambda^{1} / \omega\right) H_{1}$ and the four-nucleon contact interactions subsumed in $H_{2}$. This potential obviously agrees with the one obtained in time-dependent perturbation theory. More interesting is the first correction given in eq. (4.15). The diagrams corresponding to the various terms are shown in figs. 1-4 and 6. The first line refers to the graphs of fig. 1, the second to 1 , 2, 3 in fig. 2 and 1, 2 in fig. 6 and the third to graph 4 of fig. 2. We should mention that all graphs containing vertex corrections with exactly one $\pi \pi N N$-vertex, which are also contained in the second line, give no contributions, because only odd functions of the loop momentum enter the corresponding integrals. The first term in the fourth line subsumes graphs 5 to 8 of fig. 2 plus the irreducible self-energy diagrams depicted in fig. 3 . The next two terms in the fourth line refer to graphs 9 and 10 plus the "reducible" self-energy diagrams of fig. 4. Such "reducible" diagrams are typical for the method of unitary transformation and do not occur in old-fashioned time-ordered perturbation theory. They should not be confused with truly reducible diagrams, one example being shown in fig. 5a. In that figure, the horizontal dashed lines represent the states whose free energy enters the pertinent energy denominators. In oldfashioned time--ordered perturbation theory, such reducible diagrams are generated by iterating the potential in a Lippmann-Schwinger equation, with the potential being defined to consist only of truly irreducible diagrams. In contrast to the really reducible graphs like the one in fig. 5a, the ones resulting by applying the projection formalism do not contain the energy denominators corresponding to the propagation of nucleons only. In the same notation as used for fig. $5 \mathrm{a}$, one can e.g. express diagram 9 of fig. 2 as a sum of two graphs as depicted in fig. 5b,c. All diagrams involving four-nucleon contact interactions, shown in fig. 6, follow from the fifth line and, as already noted above, from the first term in the second line of eq. (4.15). We remark that in the c. $m$. system the three terms in the last line add up to zero. We have nevertheless made them explicit here since in time-ordered perturbation theory, these terms are treated differently and lead to the recoil correction, i.e. the explicit energy-dependence, of the two-nucleon potential. 


\subsection{Two-nucleon potential: Expressions and discussion}

As already noted above, the leading part of two nucleon potential is given by just one pion exchange with both vertices coming from eq. (C.1) and the nucleons treated as static particles plus contact interactions corresponding to eq. (C.3). This result holds in both time-dependent perturbation theory and projection formalism. For easier comparison, in this paper we use the same notation and definitions as it has been done in [⿶. In particular, the spin and isospin matrices $\vec{\sigma}$ and $\boldsymbol{t}$ satisfy the following relations:

$$
\begin{aligned}
t_{i} t_{j} & =\frac{1}{4} \delta_{i j}+\frac{1}{2} i \epsilon_{i j k} t_{k}, \\
\sigma_{i} \sigma_{j} & =\delta_{i j}+i \epsilon_{i j k} \sigma_{k} .
\end{aligned}
$$

The initial (final) momentum of nucleon in c. m. system is denoted by $\vec{p}\left(\vec{p}^{\prime}\right)$. The transferred and average momenta are given by $\vec{q}=\vec{p}-\vec{p}^{\prime}$ and $\vec{k}=\left(\vec{p}+\vec{p}^{\prime}\right) / 2$, respectively. The leading order potential is then given by

$$
V^{(0)}=-\left(\frac{2 g_{A}}{F_{\pi}}\right)^{2} \boldsymbol{t}_{1} \cdot \boldsymbol{t}_{2} \frac{\left(\vec{\sigma}_{1} \cdot \vec{q}\right)\left(\vec{\sigma}_{2} \cdot \vec{q}\right)}{\vec{q}^{2}+m_{\pi}^{2}}+C_{S}+C_{T} \vec{\sigma}_{1} \cdot \vec{\sigma}_{2}
$$

The first corrections to this result appear at order two. We now enumerate all such corrections. We start with the ones obtained using old-fashioned time-dependent perturbation theory [3] [4]. First, one seemingly obtains the contributions of tree diagrams 1 and 2 in fig. 1 with one vertex coming from eq. (C.5) and still using the static approximation for the nucleons,

$$
V_{1 \pi, \text { tree }}^{(2)}=-\frac{2 g_{A}}{F_{\pi}^{2}} \boldsymbol{t}_{1} \cdot \boldsymbol{t}_{2} \frac{\left(\vec{q} \cdot \vec{\sigma}_{1}\right)\left(\vec{q} \cdot \vec{\sigma}_{2}\right)}{\vec{q}^{2}+m_{\pi}^{2}}\left(A_{1} \vec{q}^{2}+A_{2} \vec{k}^{2}\right) .
$$

The constants $A_{1}$ and $A_{2}$ are given by

$$
A_{1}=-\left(A_{1}^{\prime}+\frac{A_{2}^{\prime}}{2}\right), \quad A_{2}=-4\left(A_{1}^{\prime}-\frac{A_{2}^{\prime}}{2}\right) .
$$

We note that these expressions differ from the ones given in appendix B of [4]. Since $\vec{k}$ and $\vec{q}$ are defined with a different prefactor as sum and difference of $\vec{p}$ and $\vec{p}^{\prime}$, there can not be the same prefactor relating the $\left(A_{1}, A_{2}\right)$ to the $\left(A_{1}^{\prime}, A_{2}^{\prime}\right)$. A few more remarks are in order: If one decomposes $\vec{q}^{2}$ as $\vec{q}^{2}+m_{\pi}^{2}-m_{\pi}^{2}$, one sees that the $A_{1}$-contribution can be completely absorbed in coupling constant and contact term redefinition. However, as discussed in appendix $\mathrm{C}$, the term (C.5) appears in the Hamiltonian with a fixed coefficient $\sim 1 / m$ representing relativistic corrections and is not relevant to the order we are working if the nucleon mass is counted via eq. (A.14). For this reason the contribution (4.19) to the two-nucleon potential can and should be completely omitted.

The contributions from tree graphs with various contact interactions from eq. (C.4) can be expressed as

$$
\begin{aligned}
V_{N N, \text { tree }}^{(2)}= & C_{1} \vec{q}^{2}+C_{2} \vec{k}^{2}+\left(C_{3} \vec{q}^{2}+C_{4} \vec{k}^{2}\right)\left(\vec{\sigma}_{1} \cdot \vec{\sigma}_{2}\right)+i C_{5} \frac{\vec{\sigma}_{1}+\vec{\sigma}_{2}}{2} \cdot(\vec{q} \times \vec{k}) \\
& +C_{6}\left(\vec{q} \cdot \vec{\sigma}_{1}\right)\left(\vec{q} \cdot \vec{\sigma}_{2}\right)+C_{7}\left(\vec{k} \cdot \vec{\sigma}_{1}\right)\left(\vec{k} \cdot \vec{\sigma}_{2}\right)
\end{aligned}
$$

where the $C_{i}$ 's are given by

$$
C_{1}=C_{1}^{\prime}-C_{3}^{\prime}+\frac{C_{2}^{\prime}}{2}
$$




$$
\begin{aligned}
& C_{2}=4 C_{1}^{\prime}-4 C_{3}^{\prime}-2 C_{2}^{\prime}, \\
& C_{3}=C_{9}^{\prime}+\frac{C_{12}^{\prime}}{2}-C_{14}^{\prime}, \\
& C_{4}=4 C_{9}^{\prime}-2 C_{12}^{\prime}+4 C_{14}^{\prime}, \\
& C_{5}=2 C_{5}^{\prime}-4 C_{4}^{\prime}-2 C_{6}^{\prime}, \\
& C_{6}=C_{7}^{\prime}+C_{8}^{\prime}+\frac{C_{10}^{\prime}}{2}+\frac{C_{11}^{\prime}}{2}-C_{13}^{\prime}, \\
& C_{7}=4 C_{7}^{\prime}+4 C_{8}^{\prime}-2 C_{10}^{\prime}-2 C_{11}^{\prime}+4 C_{13}^{\prime} .
\end{aligned}
$$

Note again that the contributions $\sim C_{5}, \sim C_{11}^{\prime}$ and $\sim C_{14}^{\prime}$ and also the signs in many places are different from what is given in [4]. Further corrections arise from irreducible one-loop diagrams 1-8 in fig. 2 :

$$
\begin{aligned}
V_{2 \pi, 1-\text { loop }, \text { irr. }=}^{(2)}= & \frac{4 g_{A}^{2}}{F_{\pi}^{4}} \int \frac{d^{3} l}{(2 \pi)^{3}} \boldsymbol{t}_{1} \cdot \boldsymbol{t}_{2} \frac{\left(\vec{l}^{2}-\vec{q}^{2}\right)}{\omega_{+} \omega_{-}\left(\omega_{+}+\omega_{-}\right)} \\
& -\frac{1}{2 F_{\pi}^{4}} \int \frac{d^{3} l}{(2 \pi)^{3}} \boldsymbol{t}_{1} \cdot \boldsymbol{t}_{2} \frac{\left(\omega_{+}-\omega_{-}\right)^{2}}{\omega_{+}+\omega_{-}} \frac{1}{\omega_{+} \omega_{-}} \\
& -\frac{g_{A}^{4}}{4 F_{\pi}^{4}} \int \frac{d^{3} l}{(2 \pi)^{3}} \frac{1}{\omega_{+}^{3} \omega_{-}}\left\{\left(\frac{3}{\omega_{-}}+\frac{8 \boldsymbol{t}_{1} \cdot \boldsymbol{t}_{2}}{\omega_{+}+\omega_{-}}\right)\left(\vec{l}^{2}-\vec{q}^{2}\right)^{2}\right. \\
& \left.+4\left(\frac{3}{\omega_{+}+\omega_{-}}+\frac{8 \boldsymbol{t}_{1} \cdot \boldsymbol{t}_{2}}{\omega_{-}}\right)\left(\vec{\sigma}_{2} \cdot[\vec{q} \times \vec{l}]\right)\left(\vec{\sigma}_{1} \cdot[\vec{q} \times \vec{l}]\right)\right\},
\end{aligned}
$$

where

$$
\omega_{ \pm} \equiv \sqrt{(\vec{q} \pm \vec{l})^{2}+4 m_{\pi}^{2}}
$$

We have also calculated the contributions from various irreducible one-loop diagrams 1-8 in fig. 3 and 1-2 in fig. 6, which involve self-energy insertions and vertex corrections. These have not been considered in [3] [4]. The corresponding contributions are given by

$$
\begin{aligned}
V_{1 \pi, 1-\text { loop, irr. }}^{(2)=} & \frac{4 g_{A}^{4}}{F_{\pi}^{4}} \int \frac{d^{3} l}{(2 \pi)^{3}} \frac{\boldsymbol{t}_{1} \cdot \boldsymbol{t}_{2}}{\omega_{l}^{2} \omega_{q}^{2}}\left\{\frac{\vec{l} \cdot \vec{q}}{\omega_{l}}\left(\left(\vec{\sigma}_{1} \cdot \vec{l}\right)\left(\vec{\sigma}_{2} \cdot \vec{q}\right)+\left(\vec{\sigma}_{1} \cdot \vec{q}\right)\left(\vec{\sigma}_{2} \cdot \vec{l}\right)\right)\right. \\
& \left.-\left(\frac{1}{\omega_{l}}+\frac{3}{\omega_{q}}\right) \vec{l}^{2}\left(\vec{\sigma}_{1} \cdot \vec{q}\right)\left(\vec{\sigma}_{2} \cdot \vec{q}\right)\right\}
\end{aligned}
$$

where

$$
\omega_{q}=\sqrt{\vec{q}^{2}+m_{\pi}^{2}}, \quad \omega_{l}=\sqrt{\vec{l}^{2}+m_{\pi}^{2}}
$$

and by

$$
\begin{aligned}
V_{N N, 1-\text { loop }, \text { irr. }}^{(2)=} & \frac{g_{A}^{2}}{F_{\pi}^{2}} C_{S} \int \frac{d^{3} l}{(2 \pi)^{3}} \frac{1}{\omega_{l}^{3}}\left\{3 \vec{l}^{2}+4\left(\boldsymbol{t}_{1} \cdot \boldsymbol{t}_{2}\right)\left(\vec{l} \cdot \vec{\sigma}_{1}\right)\left(\vec{l} \cdot \vec{\sigma}_{2}\right)\right\} \\
& +\frac{g_{A}^{2}}{F_{\pi}^{2}} C_{T} \int \frac{d^{3} l}{(2 \pi)^{3}} \frac{1}{\omega_{l}^{3}}\left\{4 \vec{l}^{2} \boldsymbol{t}_{1} \cdot \boldsymbol{t}_{2}+2\left(\vec{l} \cdot \vec{\sigma}_{1}\right)\left(\vec{l} \cdot \vec{\sigma}_{2}\right)\left(3-2 \boldsymbol{t}_{1} \cdot \boldsymbol{t}_{2}\right)\right. \\
& \left.-\vec{l}^{2}\left(\vec{\sigma}_{1} \cdot \vec{\sigma}_{2}\right)\left(3-4 \boldsymbol{t}_{1} \cdot \boldsymbol{t}_{2}\right)\right\}
\end{aligned}
$$

We remark an unpleasant feature of $V_{1 \pi, 1-\text { loop, irr. }}^{(2)}$ With $t=-\vec{q}^{2}$ one can rewrite the term proportional to $1 / \omega_{q}^{3}$ as $\left(m_{\pi}^{2}-t\right)^{-3 / 2}$. This function has a cut starting at $t=m_{\pi}^{2}$. Physically, 
this does not make sense since all cuts should be produced by multi-particle intermediate states. We come back to this later on.

Finally, the corrections arising by explicitly keeping the nucleon kinetic energy in one-pion exchange tree diagrams with both vertices from leading order Lagrangian represents the energydependent part of $2 \mathrm{~N}$-potential given by

$$
V_{E}^{(2)}=\frac{4 g_{A}^{2}}{F_{\pi}^{2}} \boldsymbol{t}_{1} \cdot \boldsymbol{t}_{2}\left(\vec{\sigma}_{1} \cdot \vec{q}\right)\left(\vec{\sigma}_{2} \cdot \vec{q}\right) \frac{E-\frac{1}{m}\left(\vec{k}^{2}+\frac{1}{4} \vec{q}^{2}\right)}{\left(\vec{q}^{2}+m_{\pi}^{2}\right)^{3 / 2}}
$$

where $E$ is an initial energy of the two nucleons (full energy). It should be stressed that onshell, this contribution vanishes. This on-shell center-of-mass kinematics is often used as an approximation for calculations of NN scattering or few-nucleon forces. However, if one iterates this potential in e.g. a Lippmann-Schwinger equation, the energy $E$ can no longer be simply related to the momenta $\vec{k}$ and $\vec{q}$. A similar comment applies to using this recoil correction in the calculation of few-nucleon forces (for $N \geq 3$ ).

Let us now look at the corrections arising in the framework of the projection formalism. The corrections from tree diagrams with one vertex taken from next-to-leading order Lagrangian do not change and are given again by eq. (4.21). Remember that we do not consider the Hamiltonian $\mathcal{H}_{3}$ given by eq. (C.5) for reasons given above and in appendix C. Consequently, there is no contribution (4.19) to the potential. Apart from the corrections from irreducible one-loop graphs 1-8 in fig. 2 given by eq. (4.23) one obtains a contributions from reducible diagrams 9 and 10, which can be expressed by

$$
\begin{aligned}
V_{2 \pi, 1-\mathrm{loop}, \mathrm{red} .}^{(2)}= & \frac{2 g_{A}^{4}}{F_{\pi}^{4}} \int \frac{d^{3} l}{(2 \pi)^{3}}\left(\frac{3}{4}-2 \boldsymbol{t}_{1} \cdot \boldsymbol{t}_{2}\right) \frac{\omega_{+}+\omega_{-}}{\omega_{+}^{3} \omega_{-}^{3}} \\
& \times\left\{\frac{1}{4}\left(\vec{l}^{2}-\vec{q}^{2}\right)^{2}-\left(\vec{\sigma}_{2} \cdot[\vec{q} \times \vec{l}]\right)\left(\vec{\sigma}_{1} \cdot[\vec{q} \times \vec{l}]\right)\right\} .
\end{aligned}
$$

Summing up the eqs. (4.23) and (4.29) we obtain the two-pion exchange contributions to the potential within the projection formalism:

$$
\begin{aligned}
V_{2 \pi, 1-\text { loop }}^{(2)}= & \frac{4 g_{A}^{2}}{F_{\pi}^{4}} \int \frac{d^{3} l}{(2 \pi)^{3}} \boldsymbol{t}_{1} \cdot \boldsymbol{t}_{2} \frac{\left(\vec{l}^{2}-\vec{q}^{2}\right)}{\omega_{+} \omega_{-}\left(\omega_{+}+\omega_{-}\right)} \\
& -\frac{1}{2 F_{\pi}^{4}} \int \frac{d^{3} l}{(2 \pi)^{3}} \boldsymbol{t}_{1} \cdot \boldsymbol{t}_{2} \frac{\left(\omega_{+}-\omega_{-}\right)^{2}}{\omega_{+}+\omega_{-}} \frac{1}{\omega_{+} \omega_{-}} \\
& -\frac{g_{A}^{4}}{F_{\pi}^{4}} \int \frac{d^{3} l}{(2 \pi)^{3}} \frac{\omega_{+}^{2}+\omega_{+} \omega_{-}+\omega_{-}^{2}}{\omega_{+}^{3} \omega_{-}^{3}\left(\omega_{+}+\omega_{-}\right)}\left\{2 \boldsymbol{t}_{1} \cdot \boldsymbol{t}_{2}\left(\vec{l}^{2}-\vec{q}^{2}\right)^{2}\right. \\
& \left.+3\left(\vec{\sigma}_{2} \cdot[\vec{q} \times \vec{l}]\right)\left(\vec{\sigma}_{1} \cdot[\vec{q} \times \vec{l}]\right)\right\} .
\end{aligned}
$$

As was first noted in ref. [20] using a different formalism, the isoscalar spin independent central and the isovector spin dependent parts of the two-nucleon potential corresponding to the twopion exchange adds up to zero. It is comforting that we find the same result. Note, that this is different from the energy-dependent potential derived using the time-ordered perturbation theory. 
The corrections arising from one-loop reducible diagrams 1-4 in fig. 4 and 3-6 in fig. 6, which involve the nucleon self-energy and loop corrections to the four-fermion interactions, are given by

$$
V_{1 \pi, 1-\text { loop, red. }}^{(2)}=\frac{12 g_{A}^{4}}{F_{\pi}^{4}} \int \frac{d^{3} l}{(2 \pi)^{3}} \boldsymbol{t}_{1} \cdot \boldsymbol{t}_{2} \frac{\omega_{l}+\omega_{q}}{\omega_{l}^{3} \omega_{q}^{3}} \vec{l}^{2}\left(\vec{\sigma}_{1} \cdot \vec{q}\right)\left(\vec{\sigma}_{2} \cdot \vec{q}\right)
$$

and by

$$
\begin{aligned}
V_{N N, 1-\text { loop, red. }}^{(2)}= & -\frac{g_{A}^{2}}{F_{\pi}^{2}} C_{S} \int \frac{d^{3} l}{(2 \pi)^{3}} \frac{1}{\omega_{l}^{3}}\left\{3 \vec{l}^{2}+4\left(\boldsymbol{t}_{1} \cdot \boldsymbol{t}_{2}\right)\left(\vec{l} \cdot \vec{\sigma}_{1}\right)\left(\vec{l} \cdot \vec{\sigma}_{2}\right)\right\} \\
& -\frac{g_{A}^{2}}{F_{\pi}^{2}} C_{T} \int \frac{d^{3} l}{(2 \pi)^{3}} \frac{1}{\omega_{l}^{3}}\left\{4 \vec{l}^{2} \boldsymbol{t}_{1} \cdot \boldsymbol{t}_{2}+4\left(\boldsymbol{t}_{1} \cdot \boldsymbol{t}_{2}\right)\left(\vec{l} \cdot \vec{\sigma}_{1}\right)\left(\vec{l} \cdot \vec{\sigma}_{2}\right)\right. \\
& \left.+\vec{l}^{2}\left(\vec{\sigma}_{1} \cdot \vec{\sigma}_{2}\right)\left(3-4 \boldsymbol{t}_{1} \cdot \boldsymbol{t}_{2}\right)\right\} .
\end{aligned}
$$

Note that $V_{1 \pi, 1-\text { loop, red. }}^{(2)}$ again contains a term $\sim 1 / \omega_{q}^{3}$. Combining eqs. 4.25) and (4.31), this unphysical contribution vanishes. Summing up the corrections eqs. (4.25), (4.27), corresponding to irreducible graphs, which are again the same as in the old-fashioned time-dependent perturbation theory and those (4.31), (4.32), corresponding to reducible diagrams, one gets the complete result, representing the one-loop contributions, which involve self-energy insertions and vertex corrections within projection formalism:

$$
\begin{aligned}
V_{1 \pi, 1-\text { loop }}^{(2)}=\frac{4 g_{A}^{4}}{F_{\pi}^{4}} \int \frac{d^{3} l}{(2 \pi)^{3}} \frac{\boldsymbol{t}_{1} \cdot \boldsymbol{t}_{2}}{\omega_{l}^{3} \omega_{q}^{2}}\left\{(\vec{l} \cdot \vec{q})\left(\left(\vec{\sigma}_{1} \cdot \vec{l}\right)\left(\vec{\sigma}_{2} \cdot \vec{q}\right)+\left(\vec{\sigma}_{1} \cdot \vec{q}\right)\left(\vec{\sigma}_{2} \cdot \vec{l}\right)\right)\right. \\
\left.+2 \vec{l}^{2}\left(\vec{\sigma}_{1} \cdot \vec{q}\right)\left(\vec{\sigma}_{2} \cdot \vec{q}\right)\right\}, \\
V_{N N, 1-\text { loop }}^{(2)}=\frac{8 g_{A}^{2}}{F_{\pi}^{2}} C_{T} \int \frac{d^{3} l}{(2 \pi)^{3}} \frac{1}{\omega_{l}^{3}}\left(\frac{3}{4}-\boldsymbol{t}_{1} \cdot \boldsymbol{t}_{2}\right)\left(\left(\vec{l} \cdot \vec{\sigma}_{1}\right)\left(\vec{l} \cdot \vec{\sigma}_{2}\right)-\vec{l}^{2}\left(\vec{\sigma}_{1} \cdot \vec{\sigma}_{2}\right)\right) .
\end{aligned}
$$

The complete potential of second order in the projection formalism is therefore given by the expressions (4.21), (4.30), (4.33) and (4.34).

We remark that one can perform the $l$-integrations in the last two equations and one finds that the contribution from eq. (4.33) can be entirely absorbed in coupling constant and wave function renormalization whereas eq. (4.34) merely amounts to the renormalization of the lowest order four-fermion contact terms. Finally, the crucial difference between the two formalisms consists in the treatment of the energy-dependent term eq. (4.28). As already noted above, it does not appear in the method of unitary transformation. We note that many of the results derived here have already been found in [20], where one- and two-pion exchange graphs were calculated by means of Feynman diagrams and using dimensional regularization. That potential can and has been applied for scattering processes but can not be used to solve the bound-state problem.

\subsection{The three-nucleon potential}

Let us consider the leading part of the three-nucleon $(3 \mathrm{~N})$-potential. For this we take a closer look at eq. (4.15). The diagram 1 in fig. 7 represents all terms in the second line of this equation, which involve the nonlinear $\pi \pi N N$ coupling. These contributions are identical in both timedependent perturbation theory and in projection formalism and, as it was noted in ref. [1], sum up to zero, when all time-orderings are taken into account. For three-nucleon forces mediated by two-pion exchange with only linear $\pi N N$ couplings one gets different results by using the 
two approaches. The irreducible graphs 1-4 in fig. 8 represent the result of old-fashioned timedependent perturbation theory. Apart from them, the reducible graphs 5 and 6 in fig. 8 occur using our approach. An exact cancellation between the contributions from those reducible and irreducible diagrams was recently pointed out in [11] for the case of an expansion in the pionnucleon coupling constant and adopting the nonrelativistic approximation for nucleons. At leading order, these two very different expansions lead to the same set of diagrams. We remark, however, that only for the method employed here one has a consistent scheme of treating the next-to-leading order corrections. Note furthermore that fig. 8 differs from the analogous one given in [11] by the topology of diagram 6. The graphs e and g shown in fig. 3 of [11] lead in general to different prefactors multiplying the energy denominators. To understand why these cancellations appear, let us take a closer look at the terms in the fourth line of eq. (4.15). We just rewrite them schematically by pulling out the common factor $M$, representing the spin, isospin and momentum structure, which is obviously the same for all of these terms. For the irreducible diagrams we then have

$$
-\left[\frac{2}{\omega_{1}\left(\omega_{1}+\omega_{2}\right) \omega_{2}}+\frac{1}{\omega_{1}^{2}\left(\omega_{1}+\omega_{2}\right)}+\frac{1}{\left(\omega_{1}+\omega_{2}\right) \omega_{2}^{2}}\right] M=-\frac{\omega_{1}+\omega_{2}}{\omega_{1}^{2} \omega_{2}^{2}} M .
$$

The contribution from the reducible diagrams can be expressed as

$$
\left[\frac{1}{\omega_{1}^{2} \omega_{2}}+\frac{1}{\omega_{1} \omega_{2}^{2}}\right] M=\frac{\omega_{1}+\omega_{2}}{\omega_{1}^{2} \omega_{2}^{2}} M
$$

The cancellation is now evident. The same sort of cancellation can be observed for diagrams 2, 3,4 in fig. 7 and for 1-loop graphs 1-6 in fig. 6 with the contact vertex $(1 / 2) C_{S}\left(N^{\dagger} N\right)\left(N^{\dagger} N\right)$ contributing to the two-nucleon potential, which correspond to the first term in the second line and the terms in the fifth line of eq. (4.15). We conclude that there is no three-nucleon force at the order $\nu=1$. This agrees with the finding of Weinberg using time-ordered perturbation theory [1]. In that case, the mechanism is different. Although the leading three-nucleon force corresponding to diagrams 2 in fig. 7 and 1-4 in fig. 8 does not vanish in that case, it was noted in [1] that these contributions cancel exactly with the energy-dependent part of the two-nucleon potential when the latter is iterated in the Lippmann-Schwinger equation. That is the reason why one can describe systems of three or more nucleons using the energy-independent part of the $2 \mathrm{~N}$-potential and without explicit three- and many-nucleon forces to the order in the chiral expansion also considered here. However, such type of cancellations does not help to remove the problems in the two-nucleon sector due to the explicit energy-dependence as noted before (i.e. that the wavefunctions are only orthonormal to the order one is working). This is the main difference to the projection approach we are using.

\section{$5 \quad$ Summary and conclusions}

In this paper, we have presented a novel approach to the problem of deriving the forces between few (two, three, ...) nucleons from effective chiral Lagrangians. For that, we use the well-known projection formalism dating back to Okubo, Fukuda, Sawada and Taketani [15] [16]. It allows to uniquely separate the effects from the operators and the wave functions. For the case at hand, we first had to modify the power counting rules since in the projection formalism one decomposes the Hilbert space of nucleons and pions into subspaces with definite nucleon and pion numbers. While in old-fashioned time-ordered perturbation theory the resulting wave functions are only orthonormal to a certain order in the chiral expansion, this problem does not 
occur in the projection formalism. Furthermore, in the previous calculations based on timeordered perturbation theory, the two-nucleon potentials turn out to be energy-dependent, which is a severe complication for applying these in systems with three or more nucleons (although to leading order, these recoil corrections are cancelled by certain $\mathrm{N}$-body interactions). Here, we have given the explicit expressions of the two-nucleon potential to two orders beyond the lowest order tree graphs (comprising the leading one-pion exchange and four-nucleon contact terms). In particular, we have also calculated self-energy type corrections and one-loop corrections to these four-fermion operators not considered before. The most salient features of the so constructed two-nucleon potential is its energy-independence and the orthonormality of the corresponding wave functions. Furthermore, we have considered the leading contributions to the three-nucleon potential. As in time-ordered perturbation theory, we find that these sum up to zero. However, the mechanism for the cancellation between some of the graphs is distinctively different in the projection formalism since it is not related to an intricate cancellation of terms generated by the iteration of the energy-dependent two-nucleon potential in old-fashioned timeordered perturbation theory. Rather, in the approach here, these cancellations can be traced back to the appearance of "reducible" graphs whose precise meaning is explained in section 4.1. These diagrams are in fact responsible for the orthonormality of the wave functions and are thus sometimes called "wave function re-orthonormalization" graphs.

Clearly, the results presented here constitute only the first step in a bigger program. We are presently working on a regularization scheme, which allows us to consistently treat the potential and the Lippmann-Schwinger equation to generate the bound state (for a recent discussion with many references to other approaches, see e.g. Beane et al. [21]). In the pioneering work by van Kolck et al. 沟, this was not done but rather a Gaussian cut-off for the momenta $\vec{q}$ and $\vec{k}$ was introduced irrespective of the power counting. Furthermore, the $\Delta(1232)$ degrees of freedom have to be incorporated in a manner analogous to what was done for the single nucleon case by Hemmert et al. [22] and finally, numerical results have to be obtained for the two- and three-nucleon systems. We believe that within the framework outlined here, one can find out to what extent chiral symmetry plays a role for the nuclear forces (the role of one- and two-pion exchanges for the higher NN partial waves was recently elucidated in [23] 20]). Furthermore, this approach can also be used to calculate meson-exchange currents in a manner which is completely consistent with the so-constructed nucleon potential.

\section{Acknowledgements}

We thank Norbert Kaiser for some very useful comments and Jamie Eden for some helpful discussions.

\section{A Chiral counting for the projected equations}

In this appendix, we work out the chiral power of the different terms appearing in eq. (3.11). Any one of these can be characterized by a counting index $\nu$, i.e. is proportional to $Q^{\nu}$. This index should not be confused with the one giving the chiral dimension of the matrix-elements $\left\langle\Psi^{i}|A| \phi\right\rangle$ discussed in the text. The free part of the Hamiltonian $H_{0}$ gives the energy, which we denote throughout by $E$. Remember that $H_{\kappa}$ denotes a term from the interaction Hamiltionian $H_{I}$ with the vertex dimension $\kappa$.

One can determine the chiral power $\nu$ for all terms entering eq. (3.11) by using of the corresponding result (3.17) for $\nu_{A}$, which, however, should be modified to take into account the 
correct number of energy denominators in each case, because of the presence of the operators $H_{0}$ and $H_{I}$. Furthermore, one uses eqs. (3.17), (3.20) to express the sum $\sum_{i} V_{i} \kappa_{i}^{A}$ over all vertices entering the operator $\lambda^{4 k+i} A_{l} \eta$ in terms of $k, i$, and $l$ as follows:

$$
\sum_{i} V_{i} \kappa_{i}^{A}=2 k+i+l
$$

For example, for $\lambda^{4 k+i} H_{\kappa} \lambda^{4 q+j} A_{l} \eta$ one has

$$
\nu=3-3 N+1-(4 k+i)+\kappa+\sum_{i} V_{i} \kappa_{i}^{A}=4-3 N-4 k-i+2 q+j+l+\kappa
$$

where $(4 k+i)$ corresponds to the number of external pion lines and we have added +1 because there is one energy denominator less than it was counted in eq. (3.17). Proceeding analogously one can derive a corresponding chiral power for all other terms in eq. (3.11), which we shall use in what follows.

Now we take a look at the possible vertices in $H_{I}$ which are explained in section 3 with $p$ pions in order to obtain few useful inequalities for determining the corresponding vertex dimension $\kappa$ :

$$
\begin{array}{ll}
\kappa \geq 2, & \text { for } p=0, \\
\kappa \geq p, & \text { for } p=1,2,3 .
\end{array}
$$

These follow from (3.18). For $p=0 n$ must be larger equal 4 and for $p=1,2,3 n$ must be greater equal 2 and there must be at least one derivative. Further

$$
\begin{aligned}
& \kappa \geq 1, \\
& \kappa \geq-2+p .
\end{aligned}
$$

Whereas (A.5) is generally valid, (A.6) follows from simple inspection of eq. (3.18): for $n=0$ there are at least two derivatives and for $n \geq 2$ it is immediately apparent.

We find the following terms with the corresponding indices $\nu$ :

- $\lambda^{4 k+i} H_{\kappa} \eta$

$$
\nu=4-3 N-4 k-i+\kappa .
$$

This follows from eq. (3.17) noting that there is one energy denominator less. It follows from (A.3), A.4), (A.6), that

$\nu \geq 4-3 N$, when $k=0$

$\nu \geq 2-3 N$, when $k>0$

- $\lambda^{4 k+i} H_{\kappa} \lambda^{4 q+j} A_{l} \eta$

$$
\nu=4-3 N-4 k-i+2 q+j+\kappa+l .
$$

The following cases are to be considered:

1. $q \leq k-1$

In this case the number of pions $p \geq 4 k+i-4 q-j$ at the vertex. Therefore the inequality (A.6) takes the form

$$
\kappa \geq-2+4 k+i-4 q-j
$$

It then follows from eq. (A.8), that

$$
\nu \geq 4-3 N-2 k \text {. }
$$


2. $q=k$

Then

$$
p \geq|j-i|
$$

We apply eq. (A.4) to this case and using the (obvious) inequality $j-i \geq-|j-i|$ one can justify eq. (A.10).

3. $q \geq k+1$

One can easily prove eq. (A.10) for this case using eq. (A.5) and by noting, that $j-i \geq-3$.

- $E\left(\lambda^{4 k+i}\right) \lambda^{4 k+i} A_{l} \eta$

$$
\nu \geq 4-3 N-2 k+l \geq 4-3 N-2 k \quad .
$$

This follows immediately from eq. (3.20) Here the first inequality becomes the exact equality when only the pion kinetic energy is taken into account in $E\left(\lambda^{4 k+i}\right)$.

- $\lambda^{4 k+i} A_{l} \eta H_{\kappa} \eta$

$$
\nu=4-3 N-2 k+l+\kappa \quad .
$$

There is no nonvanishing operator $\eta H_{\kappa} \eta$ with $\kappa<0$. That is why the inequality (A.10) is again valid.

- $\lambda^{4 k+i} A_{l} \eta E(\eta)$

We shall count the nucleon mass in the same way as it has been done by Weinberg [2]:

$$
\frac{Q}{m} \sim \frac{Q^{2}}{\Lambda_{\chi}^{2}}
$$

The nucleon kinetic energy must then be counted as $Q^{3}$ and we get the following result:

$$
\nu \geq 6-3 N-2 k+l \geq 6-3 N-2 k \text {. }
$$

- $\lambda^{4 k+i} A_{l_{1}} \eta H_{\kappa} \lambda^{4 q+j} A_{l_{2}} \eta$

$$
\nu=4-3 N-2 k+2 q+j+l_{1}+l_{2}+\kappa \geq 6-3 N-2 k .
$$

\section{B Operators at order $r$ in Eq. (3.11)}

In this appendix, we work out in detail which operators actually appear in eq. (3.11) at order $r$, which is defined in eq. (3.22). We find

- $\lambda^{4 k+i} H_{\kappa} \lambda^{4 q+j} A_{l} \eta$

One obtaines from (A.8) and (3.22) the following identity:

$$
l=r+2(k-q)+i-j-\kappa .
$$

Let us first consider the case $i \neq 0$ :

1. $q \leq k-1$

Using eqs. (A.9), (B.1) one gets

$$
l \leq r-2 k+2 q+2 \leq r .
$$


2. $q=k$

(a) $j<i$

It follows from eqs. A.11), (B.1), that

$$
l \leq r-|j-i|+i-j \leq r .
$$

(b) $j=i$

In this case eq. (B.1) takes the form

$$
l=r-\kappa \leq r-2 .
$$

due to the inequalities (A.3), A.4 and (A.6).

(c) $j \geq i+1$

The first inequality of eq. (B.3) leads to

$$
l \leq r-2 j+2 i \leq r-2 .
$$

3. $q=k+1$

(a) $i=1,2$

It can be seen from the inequalities (A.4), (A.6) that in this case $\kappa \geq 2$. Using the inequality $i-j \leq 2$ we get from eq. (B.1)

$$
l \leq r-2 \quad .
$$

(b) $i=3$

The general inequality (A.5) leads immediately to

$$
\begin{array}{lll}
\text { i. } & j=0: & l \leq r . \\
\text { ii. } & j \geq 1: & l \leq r-1 .
\end{array}
$$

4. $q \geq k+2$

Using eq. (A.6) with the number of pions $p$ given by $p=4 q+j-4 k-i$ we obtain from eq. (B.1)

$$
l \leq r-6(q-k)+2+2 i-2 j \leq r-6(q-k)+8 \leq r-4 .
$$

The case $i=0$ can be considered analogously:

1. $q \leq k-2 \Rightarrow l \leq r-2$,

2. $q=k-1$.

(a) $j \geq 1$

Putting $p=4-j$ in eq. (A.4) we see from eq. (B.1), that

$$
l \leq r-2 \text {. }
$$

(b) $j=0$

In this case we have $\kappa \geq 2$, as it follows from eq. A.6). We obtain

$$
l \leq r .
$$


3. $q=k$

It follows from eqs. (A.3) and (A.4), that

$$
l \leq r-2 .
$$

4. $q \geq k+1$

We apply the inequality (A.6) with $p=4 q+j-4 k$ to eq. (B.1) and obtain

$$
l \leq r-6(q-k)+2 \leq r-4 .
$$

- $E\left(\lambda^{4 k+i}\right) \lambda^{4 k+i} A_{l} \eta$

$$
l \leq r
$$

- $\lambda^{4 k+i} A_{l} \eta H_{\kappa} \eta$

$$
l=r-\kappa \leq r-2 .
$$

Here we have used eq. (A.3).

- $\lambda^{4 k+i} A_{l} \eta E(\eta)$

$$
l \leq r-2 .
$$

- $\lambda^{4 k+i} A_{l_{1}} \eta H_{\kappa} \lambda^{4 q+j} A_{l_{2}} \eta$

$$
l_{1}+l_{2}=r-\kappa-2 q-j \leq r-2 .
$$

\section{Explicit form of the Hamiltonian}

In this appendix we give the explicit form of interaction Hamiltonian density, which we use in this paper. It is based on the effective Lagrangian given in [4] with an important modification to be discussed below.

$$
\begin{aligned}
\mathcal{H}_{1}= & \frac{2 g_{A}}{F_{\pi}} N^{\dagger} \boldsymbol{t} \vec{\sigma} \cdot \vec{\nabla} \boldsymbol{\pi} N \\
\mathcal{H}_{2}= & \frac{2}{F_{\pi}^{2}} N^{\dagger} \boldsymbol{t} \cdot(\boldsymbol{\pi} \times \dot{\boldsymbol{\pi}}) N \\
& +\frac{1}{2} C_{T}\left(N^{\dagger} \vec{\sigma} N\right) \cdot\left(N^{\dagger} \vec{\sigma} N\right)+\frac{1}{2} C_{S}\left(N^{\dagger} N\right)\left(N^{\dagger} N\right) \\
\mathcal{H}_{4}= & C_{1}^{\prime}\left[\left(N^{\dagger} \vec{\nabla} N\right)^{2}+\left(\vec{\nabla} N^{\dagger} N\right)^{2}\right]+C_{2}^{\prime}\left(N^{\dagger} \vec{\nabla} N\right) \cdot\left(\vec{\nabla} N^{\dagger} N\right) \\
& +C_{3}^{\prime}\left(N^{\dagger} N\right)\left[N^{\dagger} \vec{\nabla}^{2} N+\vec{\nabla}^{2} N^{\dagger} N\right] \\
& +i C_{4}^{\prime}\left[\left(N^{\dagger} \vec{\nabla} N\right) \cdot\left(\vec{\nabla} N^{\dagger} \times \vec{\sigma} N\right)+\left(\vec{\nabla} N^{\dagger} N\right) \cdot\left(N^{\dagger} \vec{\sigma} \times \vec{\nabla} N\right)\right] \\
& +i C_{5}^{\prime}\left(N^{\dagger} N\right)\left(\vec{\nabla} N^{\dagger} \cdot \vec{\sigma} \times \vec{\nabla} N\right)+i C_{6}^{\prime}\left(N^{\dagger} \vec{\sigma} N\right) \cdot\left(\vec{\nabla} N^{\dagger} \times \vec{\nabla} N\right) \\
& +\left(C_{7}^{\prime} \delta_{i k} \delta_{j l}+C_{8}^{\prime} \delta_{i l} \delta_{k j}+C_{9}^{\prime} \delta_{i j} \delta_{k l}\right) \\
& \times\left[\left(N^{\dagger} \sigma_{k} \partial_{i} N\right)\left(N^{\dagger} \sigma_{l} \partial_{j} N\right)+\left(\partial_{i} N^{\dagger} \sigma_{k} N\right)\left(\partial_{j} N^{\dagger} \sigma_{l} N\right)\right] \\
& +\left(C_{10}^{\prime} \delta_{i k} \delta_{j l}+C_{11}^{\prime} \delta_{i l} \delta_{k j}+C_{12}^{\prime} \delta_{i j} \delta_{k l}\right)\left(N^{\dagger} \sigma_{k} \partial_{i} N\right)\left(\partial_{j} N^{\dagger} \sigma_{l} N\right) \\
& +\left(\frac{1}{2} C_{13}^{\prime}\left(\delta_{i k} \delta_{j l}+\delta_{i l} \delta_{k j}\right)+C_{14}^{\prime} \delta_{i j} \delta_{k l}\right) \\
& \times\left[\left(\partial_{i} N^{\dagger} \sigma_{k} \partial_{j} N\right)+\left(\partial_{j} N^{\dagger} \sigma_{k} \partial_{i} N\right)\right]\left(N^{\dagger} \sigma_{l} N\right)
\end{aligned}
$$


Here we have shown explicitly only the operators $\mathcal{H}_{\kappa}$ leading to nonvanishing $\eta H_{\kappa} \eta, \lambda^{1} H_{\kappa} \lambda^{1}$, $\lambda^{1} H_{\kappa} \eta, \lambda^{2} H_{\kappa} \eta, \lambda^{2} H_{\kappa} \lambda^{1}$ and h. c., which enter eqs. (4.9)-(4.13). The operators with three or more pion fields are irrelevant. The symbol '..' means that the appropriate products in co-ordinate and isospin space have to be taken.

Note that one has in principle four possible contact terms (two more involving $\boldsymbol{t}$ ) in eq. (C.3). However due to Fierz rearrangement they can be reduced to two. Further, the Hamiltonian used in this paper is always taken in normal ordering.

The Hamiltonian given in [4] contains apart from the terms enumerated above the two additional interactions

$$
\begin{aligned}
\mathcal{H}_{3}= & \frac{A_{1}^{\prime}}{F_{\pi}}\left[N^{\dagger}(\boldsymbol{t} \vec{\sigma} \cdot \vec{\nabla} \boldsymbol{\pi}) \vec{\nabla}^{2} N+\vec{\nabla}^{2} N^{\dagger}(\boldsymbol{t} \vec{\sigma} \cdot \vec{\nabla} \boldsymbol{\pi}) N\right] \\
& +\frac{A_{2}^{\prime}}{F_{\pi}} \vec{\nabla} N^{\dagger}(\boldsymbol{t} \vec{\sigma} \cdot \vec{\nabla} \boldsymbol{\pi}) \cdot \vec{\nabla} N,
\end{aligned}
$$

which lead to significant contributions to the two-nucleon potential at next-to-leading order. We now show that both terms (C.5) can be completely eliminated from the Hamiltonian by integrating by parts and using the nucleons' equation of motion. For this let us consider the appropriate terms in the relativistic Lagrangian which contains exactly two nucleons, one pion and three derivatives. One can express them as

$$
\left(\bar{N} a^{\mu \nu \rho}\left\{b_{1} \overleftarrow{\partial}_{\mu} \overleftarrow{\partial}_{\nu} \overleftarrow{\partial}_{\rho}+b_{2} \overleftarrow{\partial}_{\mu} \overleftarrow{\partial}_{\nu} \vec{\partial}_{\rho}+b_{3} \overleftarrow{\partial}_{\mu} \vec{\partial}_{\nu} \vec{\partial}_{\rho}+b_{4} \vec{\partial}_{\mu} \vec{\partial}_{\nu} \vec{\partial}_{\rho}\right\} \boldsymbol{t} N\right) \cdot \boldsymbol{\pi}+\text { h.c. }
$$

Note that one can easily put all other terms with one or more derivatives acting on the pion fields into the form (C.6) by integrating by parts. The most general structure of the quantity $a^{\mu \nu \rho}$ is given by

$$
\begin{aligned}
a^{\mu \nu \rho}= & a_{1} \gamma^{\mu} g^{\nu \rho}+a_{2} \gamma^{\nu} g^{\mu \rho}+a_{3} \gamma^{\rho} g^{\mu \nu}+a_{4} \gamma^{\mu} \gamma_{5} g^{\nu \rho}+a_{5} \gamma^{\nu} \gamma_{5} g^{\mu \rho}+a_{6} \gamma^{\rho} \gamma_{5} g^{\mu \nu} \\
& +a_{7} \epsilon^{\mu \nu \rho \sigma} \gamma_{\sigma}+a_{8} \epsilon^{\mu \nu \rho \sigma} \gamma_{\sigma} \gamma_{5} .
\end{aligned}
$$

Now one can see, that all terms with the totally antisymmetric tensor $\epsilon^{\mu \nu \rho \sigma}$ do not contribute because of the derivatives commute (i.e. are symmetric under interchange of the indices)

$$
\partial_{\mu} \partial_{\nu} N=\partial_{\nu} \partial_{\mu} N
$$

For all remaining terms with the metric tensor $g^{\mu \nu}$ one can make use of the equation of motion of nucleon via

$$
\left(i \gamma^{\mu} \partial_{\mu}-m+\ldots\right) N=0
$$

where the ellipsis represents terms of higher chiral dimension. We conclude that there are no corresponding terms in the relativistic Lagrangian. Nevertheless one could expect that terms of the type eq. (C.5) arise in the Lagrangian with fixed coupling constants after performing the nonrelativistic expansion for nucleons. In the one-nucleon sector such nonrelativistic expansion can be carried out explicitely using the path integral formulation heavy baryon formalism or by means of extended reparametrization invariance. One finds that one of the terms (C.5) indeed enters the Lagrangian representing a $1 / \mathrm{m}^{2}$ relativistic correction [0]. However when the nucleon mass is counted via (A.14), this term is obviously not relevant to the order we are working. Only the leading $1 / m$ correction with exactly two derivatives resulting from the Lagrangian of lowest chiral dimension and representing the nucleon kinetic energy is to be taken into account explicitely. Our conclusion about the absence of terms $(\overline{\text { C.5 }})$ in the pion-nucleon Lagrangian agrees with corresponding result in the one-nucleon sector [⿰] [24]. Finally it should be mentioned, that the structure (C.5) can not arise by going from the Lagrangian to the corresponding Hamiltonian [4]. 


\section{References}

[1] S. Weinberg, Phys. Lett. B251, 288 (1990).

[2] S. Weinberg, Nucl. Phys. B363, 3 (1991).

[3] C. Ordóñez and U. van Kolck, Phys. Lett. B291, 459 (1992).

[4] C. Ordóñez, L. Ray and U. van Kolck, Phys. Rev. Lett. 72, 1982 (1994); Phys. Rev. C53, 2086 (1996).

[5] U. van Kolck, Phys. Rev. C49, 2932 (1994).

[6] S. Weinberg, Trans. N.Y. Acad. Sci. 38, 185 (1977).

[7] V. Bernard, N. Kaiser, and Ulf-G. Meißner, Int. J. Mod. Phys. E4, 193 (1995).

[8] G. Ecker, Prog. Nucl. Part. Phys. 35, 1 (1995).

[9] C. Bloch and J. Horowitz, Nucl. Phys. 8, 91 (1958).

[10] I. Tamm, J. Phys. (USSR) 9, 449 (1945);

S.M. Dancoff, Phys. Rev. 78, 382 (1950).

[11] J.A. Eden and M.F. Gari, Phys. Rev. C53, 1510 (1986).

[12] M.F. Gari and H. Hyuga, Nucl. Phys. A278, 372 (1977);

W. Glöckle and L. Müller, Phys. Rev. C23, 1183 (1980);

S. Deister et al., Few Body Syst. 10, 1 (1991).

[13] S. Schweber, Relativistic Quantum Field Theory, Harper \& Row, 1961.

[14] R. Machleidt, K. Holinde and Ch. Elster, Phys. Rep. 149, 1 (1987).

[15] S. Okubo, Prog. Theor. Phys. 12, 603 (1954).

[16] N. Fukuda, K. Sawada and M. Taketani, Prog. Theor. Phys. 12, 156 (1954).

[17] E. Jenkins and A.V. Manohar, Phys. Lett. B255, 558 (1991).

[18] V. Bernard, N. Kaiser, J. Kambor, and Ulf-G. Meißner, Nucl. Phys. B388, 315 (1992).

[19] J. Polchinski, "Effective Field Theories and the Fermi Surface", Lectures presented at TASI 1992.

[20] N. Kaiser, R. Brockmann and W. Weise, Nucl. Phys. A625, 758 (1997).

[21] S.R. Beane, T.D. Cohen and D.R. Phillips, nucl-th/9709062, Nucl. Phys. A, in print.

[22] T.R. Hemmert, B.R. Holstein and J. Kambor, Phys. Lett. B395, 89 (1997).

[23] M.R. Robilotta and C.A. da Rocha, Nucl. Phys. A615, 391 (1997);

J.-L. Ballot,M.R. Robilotta and C.A. da Rocha, nucl-th/9801022, Phys. Rev. C, in print.

[24] G. Ecker and M. Mojžiš, Phys. Lett. B365, 312 (1996);

N. Fettes, Ulf-G. Meißner and S. Steininger, in preparation. 


\section{Figures}

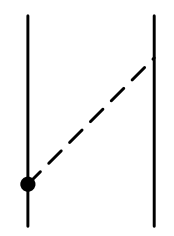

1

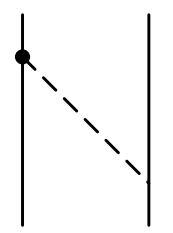

2

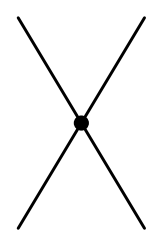

3

Figure 1: First corrections to the NN potential in the projection formalism: One-pion exchange and contact diagrams. The heavy dot denotes an insertion from the next-to-leading order pion-nucleon Lagrangian. Solid and dashed lines are nucleons and pions, respectively.

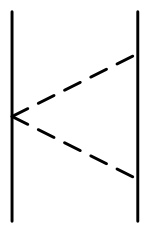

1

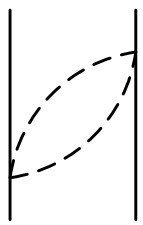

4

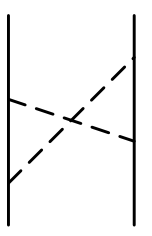

7

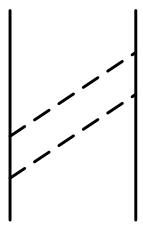

8

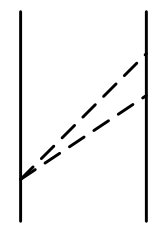

2

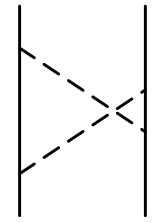

5

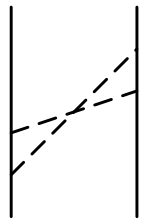

6

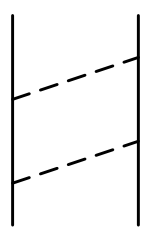

9

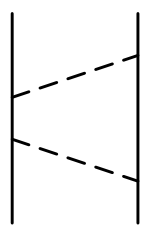

10

Figure 2: First corrections to the NN potential in the projection formalism: Two-pion exchange diagrams. Graphs which result from the interchange of the two nucleon lines are not shown. For notations, see fig. 1. 


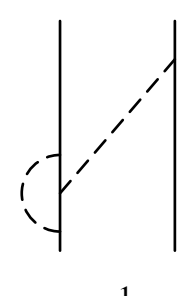

1

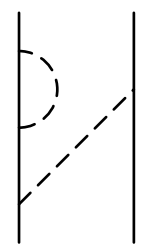

5

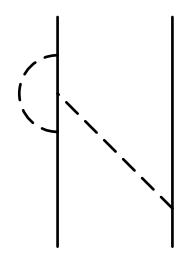

2

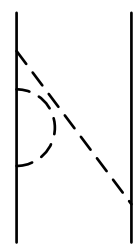

6

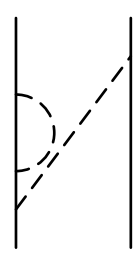

3

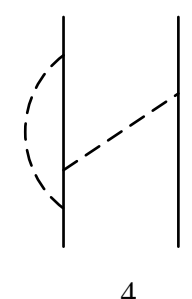

4

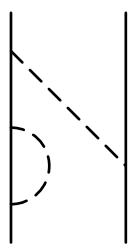

7

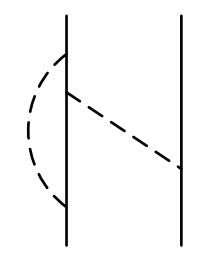

8

Figure 3: First corrections to the NN potential: Irreducible selfenergy and vertex correction graphs. For notations, see fig. 1.

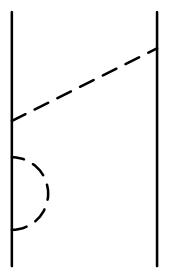

1

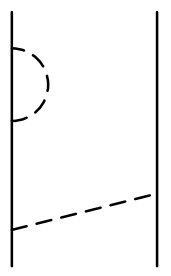

3

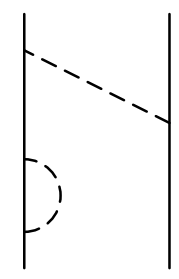

2

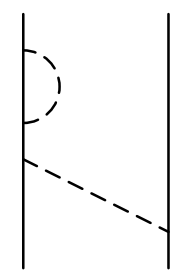

4

Figure 4: First corrections to the NN potential: Reducible selfenergy graphs. For notations, see fig. 1. 


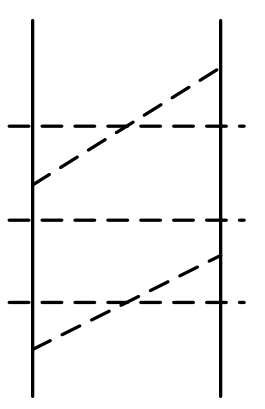

a

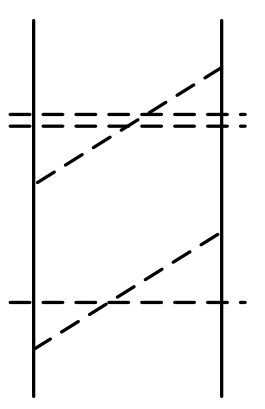

b

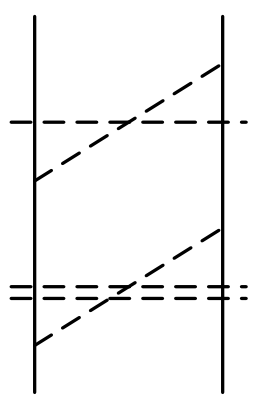

c

Figure 5: Reducible graphs. In (a), a truly reducible diagram is shown. In the projection formalism, one has graphs like (b) and (c). These correspond to diagram 9 in fig. 2. The horizontal dashed lines count the free energies of the particles cut.

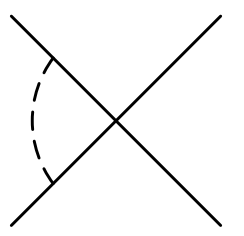

1

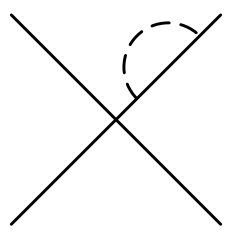

4

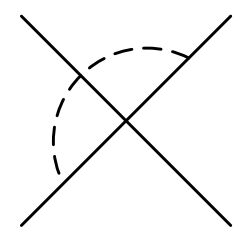

2

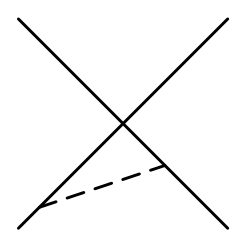

5

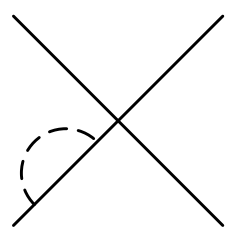

3

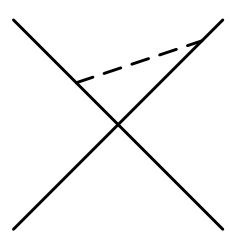

6

Figure 6: One-loop corrections to the four-nucleon contact interaction. For notations, see fig. 1. 

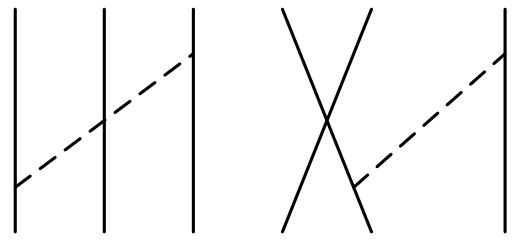

1

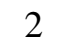

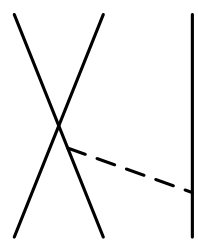

3

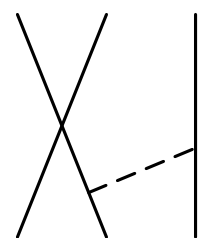

4

Figure 7: Leading diagrams contributing to the three-nucleon force that vanish. (1) is a representative for all graphs involving the $\pi \pi N N$-vertex. $(2,3,4)$ all involve one four-fermion interaction. For notations, see fig. 1.

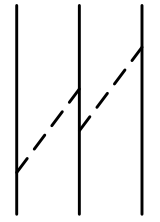

1

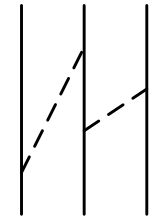

2

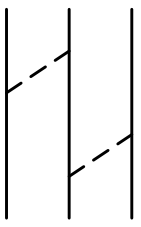

5

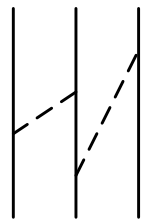

3

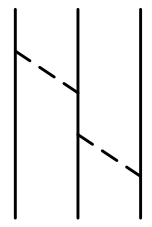

6

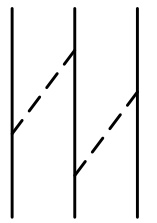

4

Figure 8: Leading diagrams contributing to the three-nucleon force. From the reducible graphs, only one representative is shown (as explained in fig. 5). For notations, see fig. 1. 